\title{
Solving a reverse supply chain design problem by improved Benders decomposition schemes ${ }^{\text {th }}$
}

\author{
Ernesto D.R. Santibanez-Gonzalez ${ }^{\mathrm{a}, *}$, Ali Diabat ${ }^{\mathrm{b}}$ \\ a Departamento de Computação, Universidade Federal de Ouro Preto, Ouro Preto, MG, Brazil \\ ${ }^{\mathrm{b}}$ Department of Engineering Systems \& Management, Masdar Institute of Science \& Technology, Abu Dhabi, UAE
}

\section{A R T I C L E I N F O}

\section{Article history:}

Received 3 October 2012

Received in revised form 11 May 2013

Accepted 6 September 2013

Available online 18 September 2013

\section{Keywords:}

Supply chain management

Reverse logistics

Benders decomposition

Mixed-integer programming

\begin{abstract}
A B S T R A C T
In this paper we propose improved Benders decomposition schemes for solving a remanufacturing supply chain design problem $(R S C P)$. We introduce a set of valid inequalities in order to improve the quality of the lower bound and also to accelerate the convergence of the classical Benders algorithm. We also derive quasi Pareto-optimal cuts for improving convergence and propose a Benders decomposition scheme to solve our RSCP problem. Computational experiments for randomly generated networks of up to 700 sourcing sites, 100 candidate sites for locating reprocessing facilities, and 50 reclamation facilities are presented. In general, according to our computational results, the Benders decomposition scheme based on the quasi Pareto-optimal cuts outperforms the classical algorithm with valid inequalities.
\end{abstract}

(c) 2013 Elsevier Ltd. All rights reserved.

\section{Introduction}

Adopting a friendly sustainable management approach implies a number of changes for companies from the strategic level up to the operational point of view, affecting their employees and impacting their business processes and technology. In this regard, Simchi-Levi, Kaminsky, and Simchi-Levi (2007) point out, "the strategic level deals with decisions that have a long-lasting effect on the firm. These include decisions regarding the number, location and capacities of warehouses and manufacturing plants, or the flow of material through the logistics network."

The problem of locating facilities and allocating customers is not new to the operations research community and involves the key aspects of supply chain design (Daskin, Snyder, \& Berger, 2005). This problem is one of "the most comprehensive strategic decision problems that need to be optimized for long-term efficient operation of the whole supply chain" (Altiparmak, Gen, Lin, \& Paksoy, 2006). As observed by Farahani and Hekmatfar (2009), some small changes to classical facility location models can make the problems much harder to solve.

In the last few years, mathematical modeling and solution methods for the efficient management of return flows (and/or integrated with forward flows) have been studied in the context of reverse logistics, closed-loop supply chains, and sustainable supply chains.

\footnotetext{
The manuscript was processed by the area editor Qiuhong Zhao.

* Corresponding author. Tel.: +55 3135591692.

E-mail address: santibanez@iceb.ufop.br (E.D.R. Santibanez-Gonzalez).
}

Fleischmann, Krikke, Dekker, and Flapper (2000) discussed new issues that arise in the context of reverse logistics, and they reviewed the mathematical models proposed in the literature. Fleischmann, Beullens, Bloemhof-Ruwaard, and Va (2001) proposed a generic recovery network model based on the elementary characteristics of return networks identified in Fleischmann et al. (2000). Zhou and Wang (2008) proposed a generic mixed integer model for the design of a reverse distribution network including repairing and remanufacturing options simultaneously.

Regarding reverse logistics networks in connection with location problems, Bloemhof-Ruwaard, Salomon, and Van Wassenhove (1996) presented a two-level distribution and waste disposal problem, in which demand for products is met by plants while the waste generated by production is correctly disposed of at waste disposal units. Barros, Dekker, and Scholten (1998) described a network for recycling sand from construction waste and proposed a two-level location model to solve the location problem of two types of intermediate facilities.

Regarding remanufacturing location models, Krikke, Van Harten, and Schuur (1999) described a small reverse logistics network for the returns, processing, and recovery of discarded copiers. They presented a mixed integer linear programming (MILP) model based on a multi-level uncapacitated warehouse location model. The model was used to determine the locations and capacities of the recovery facilities as well as the transportation links connecting various locations. In Jayaraman, Patterson, and Rolland (2003), a 0-1 MILP model for a product recall distribution problem is proposed. They analyzed a particular case in which the customer returns the product to a retail store, and the product is sent to a 
refurbishing site which will rework the product or properly dispose it. The reverse supply chain is composed of origination, collection, and refurbishing sites. With the objective to minimize fixed and distribution costs, the model has to decide which collection sites and which refurbishing sites to open, subject to a limit on the number of collection sites and refurbishing sites that can be opened.

Several authors have studied different aspects of closed-loop supply chain problems. See, for example, Jayaraman, Guide, and Srivastava (1999), Fleischmann (2003), Barbosa-Povoa, Salema, and Novais (2007), Guide and Van Wassenhove (2009) and Neto, Walther, Bloemhof-Ruwaard, Van Nunen, and Spengler (2010). Sahyouni, Savaskan, and Daskin (2007) presented three generic facility location MIP models for integrated decision making in the design of forward and reverse logistics networks. The formulations are based on the well-known uncapacitated fixed-charge location model, and they include the location of used product collection centers and the assignment of product return flows to these centers. Lu and Bostel (2007) presented a two-level location problem with three types of facilities to be located in a reverse logistics system. They proposed a $0-1$ MILP model which simultaneously considers "forward" and "reverse" flows and their mutual interactions. The model has to decide the number and locations of three different types of facilities: producers, remanufacturing centers, and intermediate centers.

In summary, reverse logistics models are chronologically discussed by Bloemhof-Ruwaard et al. (1996), Barros et al. (1998), Krikke et al. (1999), Fleischmann et al. (2000, 2001), Shih (2001), Sodhi and Reimer (2001), Jayaraman et al. (2003), Le Blanc, Fleuren, and Krikke (2004), Listeş and Dekker (2005), and more recently Zhou and Wang (2008), Salema, Barbosa-Povoa, and Novais (2010), Gomes, Barbosa-Povoa, and Novais (2011) and Alumur, Nickel, Saldanha-da-Gama, and Verter (2012). Almost all of this research proposed MILP models. Listeş and Dekker proposed a stochastic MILP and Alumur et al. studied the dynamic factors that affect location decisions. The majority of solution methods are based on standard commercial packages.

In this paper, the problem of designing a reverse supply chain network is addressed, and a Benders decomposition-based algorithm is proposed for solving it. The problem is a NP-hard combinatorial optimization problem. The MILP model follows some previously published work by, for example, Jayaraman et al. (2003), Salema et al. (2010) and Li (2011), but in this paper we develop a more efficient algorithm for solving it. For randomly generated test instances of the problem, we analyze the performance of the algorithms in term of computational times and quality of the solution obtained.

This paper makes three primary contributions. First, it proposes a Benders decomposition algorithm for solving large-scale reverse network design problems. We test two sets of valid inequalities to strengthen the Relaxed Master Problem in order to improve the quality of the lower bound. Second, we derive quasi Pareto-optimal cuts as another strategy to accelerate convergence, and third, we propose a primal based Benders decomposition algorithm for solving the problem. Computational results are conducted on largescale networks of up to 700 sourcing facilities, 100 candidate sites for locating reprocessing facilities, and 50 reclamation facilities $(700 \times 100 \times 50)$. To the best of our knowledge, for this kind of problems, these are the largest problems explored and solved so far.

The remainder of this paper is organized as follows. In the second section, we formulate the mathematical model for the problem. In the third section, the Benders decomposition-based algorithms for solving the problem are proposed. In the fourth section, we present some strategies for accelerating the convergence of the Benders algorithms. In the fifth section, the computational experiments performed with the algorithms are presented. The last section contains our conclusions.

\section{Model for designing a reverse supply chain network}

The problem can be categorized as a single product, static, three-echelon, capacitated location model with known demand. The reverse supply chain network consists of three types of members: sourcing facilities (origination sites like a retail store), collection sites, and reclamation facilities. At the customer levels, there are product demands and used products ready to be recovered (for example, cell phones). We suppose that customers return products to origination sites like a retail store. At the second layer of the supply chain network, there are reprocessing centers (collection sites) used only in the reverse channel, and they are responsible for activities such as cleaning, disassembly, checking, and sorting, before the returned products are sent back to reclamation facilities. At the third layer, reclamation facilities accept the checked returns from intermediate facilities and they are responsible for the process of reclamation. In this paper, we address the backward flow of returns coming from sourcing facilities and going to reclamation facilities through reprocessing facilities properly located at pre-defined sites. In such a supply chain network, the "reverse" flow moves from customers through collection sites to reclamation facilities and is formed by used products. On the other hand, the "forward" flow moves from reclamation facilities directly to new products at points of sale.

\subsection{RSCP model}

In this model, it is assumed that new product demands and available quantities of used products are known and deterministic. All returned (used) products are first shipped back to collection facilities where some of them will be discarded for various reasons, including poor quality. The checked return-products will then be sent back to reclamation facilities, where some of them may still be discarded. We introduce the following inputs and sets:

$I$ the set of sourcing facilities at the first layer, indexed by $i$

$J$ the set of reclamation nodes at the third layer indexed by $j$

$K$ the set of candidate reprocessing facility locations at the mid layer, indexed by $k$

$a_{i} \quad$ supply quantity at source location $i \in I$

$b_{j}$ demand quantity at reclamation location $j \in J$

$f_{k} \quad$ fixed cost of locating a mid layer reprocessing facility at candidate site $k \in K$

$g_{k} \quad$ management cost at a mid layer reprocessing facility at candidate site $k \in K$

$c_{i k}$ is the unit cost of delivering products at $k \in K$ from a source facility located in $i \in I$

$d_{k j}$ is the unit cost of supplying demand $j \in J$ from a mid layer facility located in $k \in K$

$m_{k}$ capacity at reprocessing facility location $k \in K$

We consider the following decision variables:

$w_{k} \quad 1$ if we locate a reprocessing facility at candidate site $k \in K, 0$ otherwise

$x_{i k} \quad$ flow from source facility $i \in I$ to reprocessing facility located at $k \in K$

$y_{k j}$ flow from reprocessing facility located at $k \in K$ to facility $j \in J$

Following the model proposed by Li (2011), the reverse supply chain design problem $(R S C P)$ is defined by: 


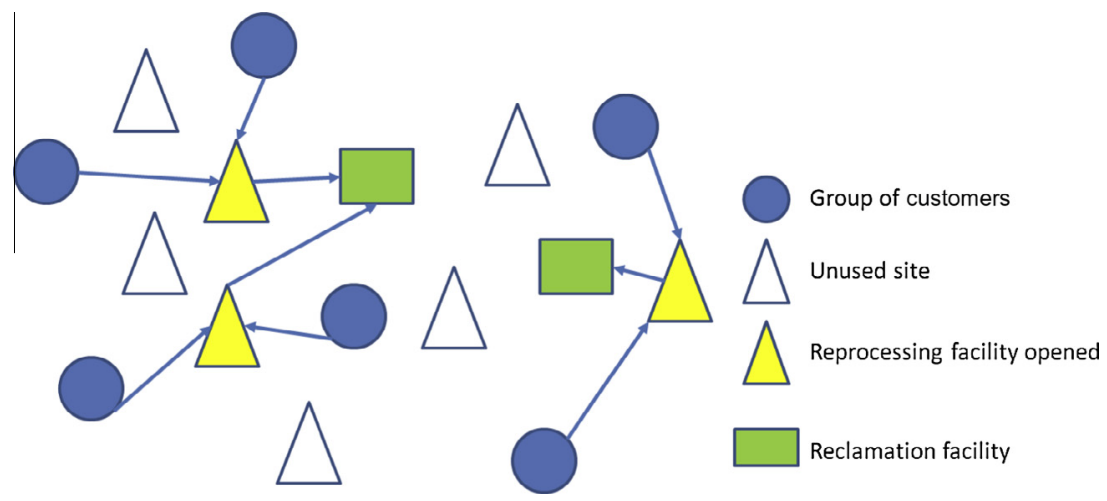

Fig. 1. Reverse supply chain network.

$$
\begin{aligned}
\text { Minimize } & \sum_{k \in K} f_{k} w_{k}+\sum_{i \in I} \sum_{k \in K}\left(c_{i k}+g_{k}\right) x_{i k}+\sum_{k \in K} \sum_{j \in J} d_{k j} y_{k j} \\
& \sum_{i \in I} x_{i k} \leqslant m_{k} w_{k} \quad \forall k \in K \\
& \sum_{k \in K} x_{i k} \leqslant a_{i} \quad \forall i \in I \\
& \sum_{k \in K} y_{k j} \geqslant b_{j} \quad \forall j \in J \\
& \sum_{i \in I} x_{i k}=\sum_{j J} y_{k j} \quad \forall k \in K \\
& x_{i k}, y_{k j} \geqslant 0, \quad \forall i \in I, \forall j \in J, \forall k \in K \\
& w_{k} \in\{0,1\} \quad \forall k \in K
\end{aligned}
$$

The objective function (1) minimizes the sum of the installation reprocessing facility costs plus management costs at the reprocessing facilities plus delivery costs from sourcing facilities to reprocessing facilities and from these to reclamation facilities. Constraints (2) ensure that all of the products that arrive at reprocessing site $k \in K$ must be less than its capacity and that this facility must be opened. Constraints (3) guarantee that up to a volume of $a_{i}$ return products available at $i$ are going backward to facility $k \in K$. Constraints (4) warrant that the demand at facility $j \in J$ must be satisfied by reprocessing facilities. Constraints (5) ensure that all the return products arriving to facility $k$ are also delivered to reclamation facilities. Constraints (6) are the standard nonnegative constraints. Constraints (7) are the standard binary constraints. This model has $O\left(n^{2}\right)$ continuous variables where $n=\max \{|I|,|J|,|K|\}$ and $|K|$ are binary variables. The number of constraints is $O(n)$.

Fig. 1 shows a reverse supply chain network consisting of 6 groups of customers, 8 candidate sites for locating reprocessing facilities (with three facilities opened), and two reclamation facilities.

\section{Benders decomposition}

Benders decomposition is a classical decomposition technique (Benders, 1962) which is advisable for solving hard mixed integer programming problems with integer variables and coupling constraints. Benders decomposition technique involves decomposing the overall formulation into a Master problem and a (Benders) subproblem, and then solving them iteratively by utilizing the solution of one in the other. The Benders Master problem with only integer variables and an auxiliary variable is a relaxation of the primary problem. The auxiliary variable is introduced to facilitate the interaction between the Master problem and the subproblem. In a minimization problem, an optimal solution of the Benders Master problem provides a lower bound on the original problem and the values of the integer variables are passed through the subproblem for solving a new subproblem. By fixing the integer variables, the primary problem with only continuous variables becomes a Benders subproblem that can be solved easily. By solving the subproblem, the values of its dual variables and a valid upper bound for the primary problem (in the case of minimization) are obtained, and, in this case, by utilizing its objective value along with the cost components implied by the Master problem solution. The bounds are updated, and if the stopping criterion is not met, a Benders cut using the dual subproblem solution is generated. This new cut is appended to the Master problem, and the Benders decomposition algorithm is iterated continuously until a stopping criterion is met. To establish a stopping criteria, we employ a small percentage gap between the best upper and lower bounds and a maximum number of Benders cycles reached; whatever is achieved first ends the algorithm.

There are a number of publications to support the fact that the Benders algorithm has not been uniformly successful in all applications; it has some deficiencies. Geoffrion and Graves (1974), among others, have noted that reformulating a mixed integer program can have a profound effect upon the efficiency of the algorithm. For the particular case of a network design problem (Magnanti \& Wong, 1981), it has been observed that a straightforward implementation of the Benders algorithm often converged very slowly, requiring the solution of a large number of Relaxed Master Problems. The main issues associated with slow convergence of Benders algorithms are (a) the solution of Relaxed Master Problems and Benders subproblems, and (b) the quality of the cuts produced in each iteration. To overcome these issues, accelerating techniques have been studied by a number of authors, among them: McDaniel and Devine (1977), Magnanti and Wong (1981), Côté and Laughton (1984), Papadakos (2008), Rei, Cordeau, Gendreau, and Soriano (2008), Saharidis, Minoux, and Ierapetritou (2010), Sherali and Lunday (2011) and Tang, Jiang, and Saharidis (2012). In a seminal paper, Magnanti and Wong (1981) proposed generating more than one cut in each iteration to accelerate the Benders algorithm, using what they refer to as Pareto-optimal cuts. A cut is defined as Pareto-optimal if no other cut dominates it. The authors propose to add, in each iteration of the algorithm, two types of cuts: the optimality or feasibility cut produced by the classical Benders procedure, and also the Pareto-optimal cuts. The results obtained by their approach showed a significant improvement in the algorithm convergence.

In the context of facility location problems, the Benders decomposition algorithm has been successfully applied by many researchers. Among them, Geoffrion and Graves (1974) employed the Benders decomposition algorithm to solve the two-stage distribution system design problem. Dogan and Goetschalckx (1999) studied the integrated design problem of a multi-period production-distribution system. Cordeau, Pasin, and Solomon (2006) solved an integrated model for a logistics network design problem, and Wentges (1996) used Pareto-optimal cuts and a procedure to 
strengthen Benders cuts to speed up the Benders decomposition algorithm for a discrete capacitated facility location problem.

In this paper, we will use Benders decomposition algorithms to solve a reverse supply chain design problem. We derive a series of valid inequalities (VI) to add to the Benders Master problem to restrict its solution space. We enter these VIs into a classical Benders decomposition algorithm to solve the problem. We also propose a Pareto-optimal cut strategy to generate cuts and we test it in order to accelerate the convergence and to evaluate the impact on the quality of the bounds obtained. Regarding the Pareto-optimal strategy, we propose to use a primal-dual Benders decomposition algorithm to generate quasi Pareto-optimal cuts and to solve the problem. The algorithms are compared in terms of speed of convergence and quality of the bounds generated.

\subsection{Decomposition scheme}

Consider the special structure of our formulation that facilitates a decomposition approach. Notice that the integer variables, $w$, and the flows variables, $x$ and $y$, are related decisions and that constraint (2) is a coupling constraint. We observe that by fixing the number and location of facilities, i.e., for fixed $w$ values, we get a linear program that can be solved efficiently. Utilizing this structure, in our Benders decomposition based solution algorithm, the Master problem includes the binary integer location decisions and the subproblem determines the flows of return products from the sourcing sites to reclamation facilities throughout reprocessing facilities already opened.

To simplify, consider the following mixed integer linear programming Principal Problem (PP), where the vector $y$ contains a number of facility location variables which are considered complicated variables, while vector $x$ contains a number of return flows positive variables.

$(P P)$

$$
\begin{aligned}
\text { Minimize } & c_{1}^{T} x+c_{2}^{T} y \\
\text { Subject to } & A x+B y \leqslant b \\
& x \geqslant 0 \\
& y \in\{0,1\}
\end{aligned}
$$

Applying Benders decomposition to this problem, the decision variables are partitioned into two sets: $x$ and $y$, and the principal problem is decomposed into the Relaxed Master Problem (RMP) and a series of Benders subproblems (BSP).

Fixing integer variables $y=\ddot{y}$ in $\mathrm{PP}$, the general form of the Benders subproblem is as follows:

(BSP)

$$
\begin{aligned}
\text { Minimize } & c_{1}^{T} x \\
\text { Subject to } & A x \leqslant b-B \ddot{y} \\
& x \geqslant 0
\end{aligned}
$$

The dual problem of BSP (D-BSP) can be written as $(D-B S P)$

$$
\begin{array}{ll}
\text { Maximize } & (b-B \ddot{y})^{T} u \\
\text { Subject to } & A^{T} u \leqslant c_{1}^{T} \\
& u \leqslant 0
\end{array}
$$

And the general form of the Master Problem (MP) is as follows: (MP)

$$
\begin{aligned}
\text { Minimize } & c_{1}^{T} x+z \\
\text { Subject to } & (b-B \ddot{y})^{T}\left(u^{p}\right) \leqslant z \\
& (b-B \ddot{y})^{T}\left(u^{q}\right) \leqslant 0 \\
& y \in\{0,1\}
\end{aligned}
$$

where $u^{p}$ and $u^{q}$ are vectors of extreme points and extreme rays, respectively, of the polyhedron formed by constraints (16) and (17), and $z$ is an auxiliary continuous variable. The main drawback of MP is that the dimension of vectors $u^{p}$ and $u^{q}$ is usually extremely large. To overcome this limitation, the Benders algorithm proposes to generate constraints (19) and (20) iteratively, as we will explain later in this paper.

Following the Benders decomposition scheme described above, for our RSCP problem, we obtain the following problems:

\subsection{Subproblem}

By fixing binary (facility location) variables $w_{k}=\ddot{w}_{k}$, the primal Benders subproblem (PBS) in our RSCP problem is as follows:

(PBS)

$$
\begin{array}{ll}
\text { Minimize } & \sum_{i \in I} \sum_{k \in K}\left(c_{i k}+g_{k}\right) x_{i k}+\sum_{k \in K} \sum_{j \in J} d_{k j} y_{k j} \\
\text { Subject to } & \sum_{i \in I} x_{i k} \leqslant m_{k} \ddot{w}_{k} \quad \forall k \in K \\
& \sum_{k \in K} x_{i k} \leqslant a_{i} \quad \forall i \in I \\
& \sum_{k \in K} y_{k j} \geqslant b_{j} \quad \forall j \in J \\
& \sum_{i \in I} x_{i k} \geqslant \sum_{j \in J} y_{k j} \quad \forall k \in K \\
& x_{i k}, y_{k j} \geqslant 0, \quad \forall i \in I, \forall j \in J, \forall k \in K
\end{array}
$$

Notice that, without loss of generality, constraint (26) replaces constraint (5), considering that,

$\sum_{i \in I} a_{i} \geqslant \sum_{j \in J} b_{j}$

Defining dual variables $u_{k}$ associated with constraint (23), $s_{i}$ associated with constraint (24), associated with constraint (25), and $q_{k}$ associated with constraint (26), we can write the dual of the PBS problem (DBS) as follows:

(DBS)

$$
\begin{array}{cc}
\text { Maximize } & \sum_{j \in J} b_{j} r_{j}-\left(\sum_{k \in K} m_{k} \ddot{w}_{k} u_{k}+\sum_{i \in I} a_{i} s_{i}\right) \\
\text { Subject to } & q_{k}-s_{i}-u_{k} \geqslant 0, \quad \forall i \in I, \forall k \in K \\
& q_{k}-r_{j} \geqslant 0, \quad \forall j \in J, \forall k \in K \\
& q_{k}, r_{j}, s_{i}, u_{k} \geqslant 0, \quad \forall i \in I, \forall j \in J, \forall k \in K
\end{array}
$$

By duality theory, if the PBS problem is infeasible for the fixed values $\ddot{w}_{k}$, then the DBS problem has a bounded solution which is an extreme point of the polyhedron $\Omega$ constituted by (30)-(32), and thus an optimality Benders cut is deduced, taking the form in (33). If the PBS problem is infeasible, the DBS problem has an unbounded solution, an extreme ray of the polyhedron can be identified, and a feasibility Benders cut will be generated taking the form in (34).

Let $P \Omega$ and $Q \Omega$ be the sets of all the extreme points and extreme rays of polyhedron $\Omega$, respectively; $z$ an auxiliary variable, then the optimality and feasibility Benders cuts are as follows:

$z \geqslant \sum_{j \in J} b_{j} r_{j}^{p}-\left(\sum_{k \in K} m_{k} \ddot{w}_{k} u_{k}^{p}+\sum_{i \in I} a_{i} s_{i}^{p}\right)$

and

$0 \geqslant \sum_{j \in J} b_{j} r_{j}^{Q}-\left(\sum_{k \in K} m_{k} \ddot{w}_{k} u_{k}^{Q}+\sum_{i \in I} a_{i} s_{i}^{Q}\right)$

where $r_{j}^{p}, s_{i}^{p} a n d u_{k}^{p} P_{\Omega}$ and $r_{j}^{Q}, s_{i}^{Q}$ and $u_{k}^{Q} Q_{\Omega}$. 
Set initial feasible $\ddot{w}_{k}, z_{l b}=-\infty, z_{u b}=+\infty$

While No convergence do

Solve $D B S$ subproblem

If solved to optimality

Optimality cut: Get extreme point of type (36)

Update $z_{u b}$

Else if Unbounded

Feasibility cut: Get extreme ray of type (37)

End if

Add cut(s) to $R M P$

Solve $R M P$ with new cuts

Update $z_{l b}$

End while

Fig. 2. Pseudo-code for a classical Benders decomposition algorithm.

\subsection{Relaxed Master Problem}

Based on the cuts of type (33) and (34) described above, the Relaxed Master Problem (RMP) in our RSCP can be written as follows: (RMP)

Minimize $\sum_{k \in K} f_{k} w_{k}+z$

Subject to

$z \geqslant \sum_{j \in J} b_{j} r_{j}^{p}-\left(\sum_{k \in K} m_{k} \ddot{w}_{k} u_{k}^{p}+\sum_{i \in I} a_{i} s_{i}^{p}\right)$

$0 \geqslant \sum_{j \in J} b_{j} r_{j}^{Q}-\left(\sum_{k \in K} m_{k} \ddot{w}_{k} u_{k}^{Q}+\sum_{i \in I} a_{i} s_{i}^{Q}\right)$

$w_{k} \in\{0,1\} \quad \forall k \in K$

The optimality Benders cuts (36) can strengthen the lower bound obtained from the RMP problem while the feasibility Benders cuts (37) make the lower bound valid for the $R S C P$ primary problem.

\subsection{Benders decomposition algorithm}

In Fig. 2 we show the pseudo-code of a classical Benders decomposition algorithm. We use this scheme jointly with the valid inequalities described in the next section.

\section{Strategies for accelerating Benders decomposition algorithm}

\subsection{Valid inequalities}

We derive a series of valid inequalities to strengthen the Master problem and to improve the lower bound provided by the Benders decomposition algorithm. As described earlier in this paper, one of the reasons for slow convergence of the Benders decomposition algorithm is that the lower bound provided by the RMP problem could be relatively weak.

According to the structure of our problem, we developed the following valid inequality (VI) to narrow the solution space of the RMP:

(1) Forcing to open at less than one facility - wcon
Tragantalerngsak, Holt, and Rönnqvist (2000) proposed to improve the relaxation of a related capacitated location problem by introducing a constraint of the following type:

$\sum_{k \in K} w_{k} \geqslant 1$

Constraint (39) forces the selection of at least one facility to be open.

(2) Feasibility - facilities servicing the demand - wcon1

$\sum_{k \in K} m_{k} \ddot{w}_{k} \geqslant \sum_{j \in J} b_{j}$

Constraint (40) ensures that opened reprocessing facilities have enough capacity to service all the demand for returned products at reclamation facilities.

\subsection{Generation of Pareto-optimal cuts}

The BSP problem has a network structure; hence, it typically has multiple dual solutions as an alternative for the optimality cut computed by (36). We would like to generate a cut that dominates others' cuts in the vicinity of the optimal solution. According to Magnanti and Wong (1981), a Pareto-optimal cut can be obtained by solving the following problem:

$(P O-B S P)$

$$
\begin{array}{ll}
\text { Maximize } & \left(b-B y^{+}\right)^{T} u \\
\text { Subject to } & A^{T} u \leqslant c_{1}^{T} \\
& (b-B \ddot{y})^{T} u \leqslant(b-B \ddot{y})^{T} u^{*} \\
& u \leqslant 0
\end{array}
$$

where $(b-B \ddot{y})^{T} u^{*}$ is the optimal objective value of the dual subproblem $(D-B S P)$ and $y^{+}$is a core point of the solution space of the Relaxed Master problem (RMP).

For our problem, we use the following two facts for deriving Pareto-optimal cuts:

- any extreme point or any extreme ray of the dual subproblem gives a valid Benders cut (Benders, 1962);

- it is not necessary to use a core point of the solution space of the Master problem to produce a Pareto cut (Papadakos, 2008).

Then, the following auxiliary problem can produce Pareto-optimal cuts:

(APO)

$$
\begin{array}{ll}
\text { Maximize } & \left(b-B y^{-}\right)^{T} u \\
\text { Subject to } & A^{T} u \leqslant c_{1}^{T} \\
& (b-B \ddot{y})^{T} \leqslant c_{1}^{T} x^{*} \\
& u \leqslant 0
\end{array}
$$

where $c_{1}^{T} x^{*}$ is the optimal objective value of the primal subproblem (BSP) and $y^{-}$is the linear relaxation solution of the Master problem.

Fig. 3 depicts the pseudo-code of a classical Benders decomposition algorithm considering the procedure for generating Pareto-optimal cuts (Cordeau et al., 2006; Magnanti \& Wong, 1981; Papadakos, 2008). Observe that it includes the auxiliary problem solution for obtaining the Pareto-optimal cuts, after solving the DSP problem. That is because it used the optimal solution of the DSP for generating the optimality cut to be included in the Master problem. As explained before, the core point is obtained by getting the LP solution of the Master problem.

\section{Computational experiments}

In this section we describe computational experiments using the proposed Benders decomposition methods for solving 


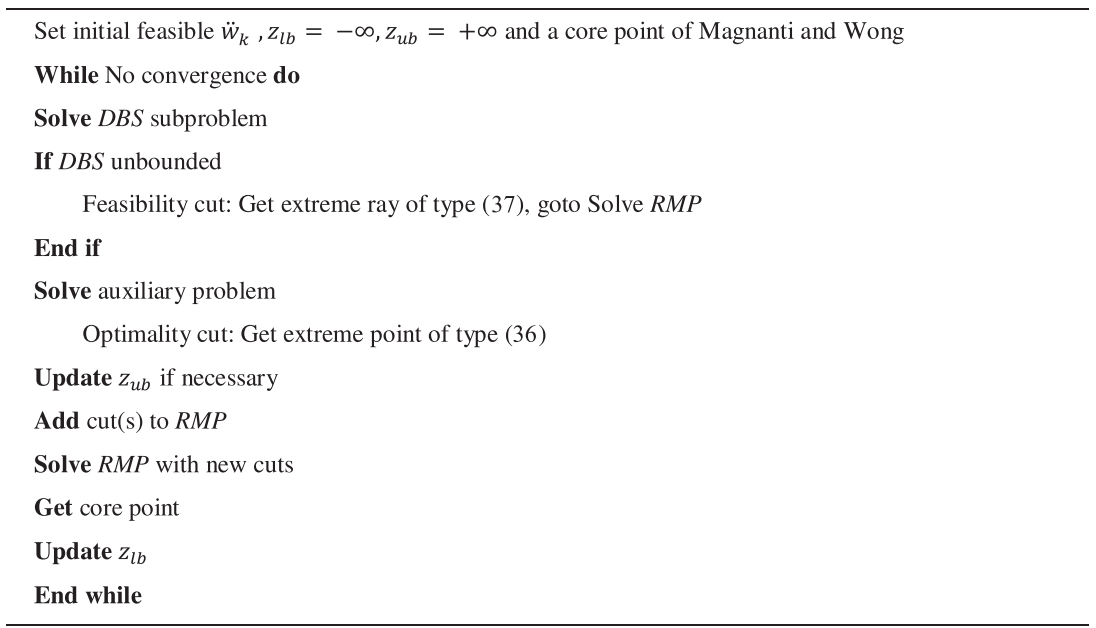

Fig. 3. Pseudo-code for a classical Benders decomposition algorithm with Pareto-optimal cuts.

large-scale reverse supply chain design problems. We first describe the characteristics of the test problems and some implementation details, and then we demonstrate the computational efficiencies achieved by each of the Benders decomposition methods in terms of computing times, quality of the lower and upper bounds, and the number of Benders cycles.

To evaluate the impact of employing the different proposed valid inequalities (VI), we solve each instance with our Benders algorithm, given in Fig. 1, both with and without the proposed VI. The effects of the different types of cuts on the performance of the Benders decomposition algorithm are compared and the computational results are reported. The Benders decomposition algorithms are coded in GAMS and tested on the computer with CPU of 2.3 GHz and 4.0 GB RAM. Whenever Benders decomposition algorithms are needed to solve the Master problem and Benders subproblems, CPLEX (version 12.3) with default settings is used as an optimization solver.

The Benders decomposition algorithms stopped when one of the following criteria was reached:

(i) the optimality gap $\left(100 *\left(z_{u b}-z_{l b}\right) / z_{l b}\right)$ was below a threshold value $\varepsilon=0.009$;

(ii) the maximum number of iterations (Benders cycles) was hit: we set it to 50 .

For the alternative types of VI and Pareto-optimal cuts, the progression of $z_{u b}$ and $z_{l b}$ values over the iterations is depicted in Fig. 4. For space limitation, we plot the results for a problem instance of $600 \times 100 \times 40$. We can observe that the use of proposed VI of type wcon 1 is effective in speeding up convergence and that the quality of both the upper and lower bounds is affected. We also observe the same effects when using the Pareto optimal cuts. Note that, in the case of the Pareto-optimal cuts, we developed an alternative Benders decomposition algorithm based on the solution of a primal subproblem. Fig. 4 represents what we observe in our numerical studies with other instances: a similar high performance in convergence with cuts and, in particular, with a VI of type wcon1. We observe that alternative Benders decomposition algorithms with Pareto optimal cuts converge very quickly to the best lower bound.

\subsection{Data generation}

We randomly generated a set of 10 instances ranging from networks of 350 sourcing facilities, 100 candidate sites for locating reprocessing facilities, and 40 reclamation facilities $(350 \times 100 \times 40)$ up to $700 \times 100 \times 40$. The size of an instance var-

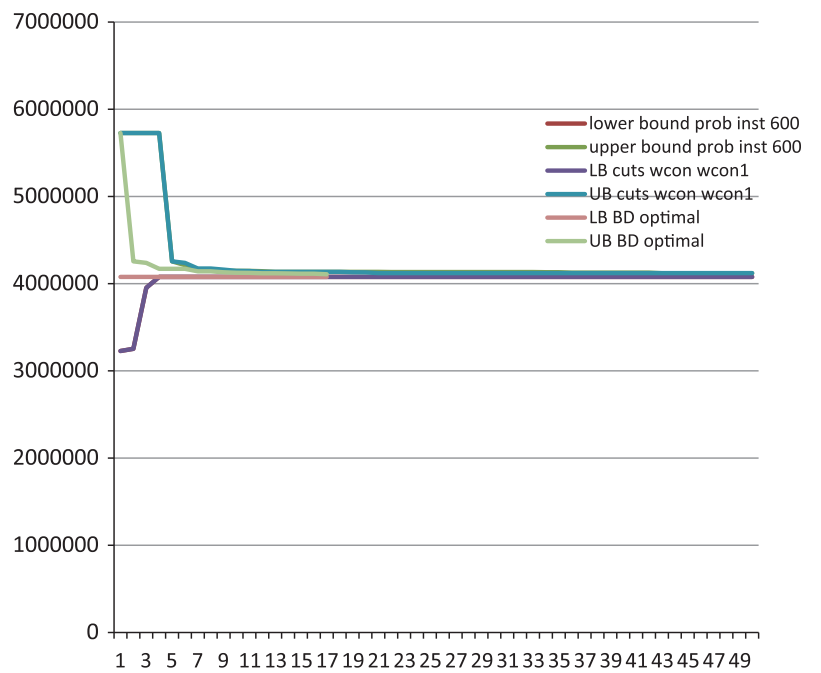

Fig. 4. Upper and lower bounds provided by the different Benders algorithms for problem instance 9 .

ies according to the number of sourcing facilities $(|I|)$, the number of candidate sites for locating reprocessing facilities $(|K|)$, and the number of reclamation facilities $(|J|)$. The data set for the test problems is given in Table 1. All the transportation costs were generated randomly using a uniform distribution with parameters $[1,40]$. Management costs $\left(g_{k}\right)$ were set to 30 for all problem instances. Fixed costs $\left(f_{k}\right)$ for problem instances $6-10$ were obtained, multiplying by 10 the fixed costs of the corresponding reprocessing facilities of problem instances $1-5$. Sourcing units $\left(a_{i}\right)$, the capacity of reprocessing facilities $\left(m_{k}\right)$, and the capacity of reclamation facilities $\left(b_{j}\right)$ are shown in Table 1.

To illustrate the size differences between the problem instances of the RSCP model, for problem instances $1-5$, Table 2 shows the number of continuous and binary variables as well as the number of constraints.

\subsection{Results analysis}

In Table 3, the lower and upper bounds, gap (\%), and number of Benders cycles are presented for Benders algorithms without any additional VI and with VI of type wcon. These VI do not produce any significant impact, neither on the gap nor in the number of Benders cycles. To illustrate, for problem instance 3, gaps and 
Table 1

Data set.

\begin{tabular}{rllllllrl}
\hline \multicolumn{1}{c}{$\#$} & Instance problems & $|I|$ & $|K|$ & $|J|$ & \multicolumn{1}{l}{$f_{k}$} & $a_{i}$ & \multicolumn{1}{c}{$m_{k}$} & \multicolumn{1}{l}{$b_{j}$} \\
\hline 1 & $350 \times 100 \times 40$ & 350 & 100 & 40 & 2000 & 200 & 800 & 1750 \\
2 & $400 \times 100 \times 40$ & 400 & 100 & 40 & 2000 & 200 & 1500 & 2000 \\
3 & $500 \times 100 \times 40$ & 500 & 100 & 40 & 2000 & 200 & 1500 & 2500 \\
4 & $600 \times 100 \times 40$ & 600 & 100 & 40 & 2500 & 300 & 3000 & 2500 \\
5 & $700 \times 100 \times 40$ & 700 & 100 & 40 & 3000 & 300 & 3000 & 2500 \\
6 & $350 \times 100 \times 40$ & 350 & 100 & 40 & 20,000 & 200 & 800 & 1750 \\
7 & $400 \times 100 \times 40$ & 400 & 100 & 40 & 20,000 & 200 & 1500 & 2000 \\
8 & $500 \times 100 \times 40$ & 500 & 100 & 40 & 20,000 & 200 & 1500 & 2500 \\
9 & $600 \times 100 \times 40$ & 600 & 100 & 40 & 25,000 & 300 & 3000 & 2500 \\
10 & $700 \times 100 \times 40$ & 700 & 100 & 40 & 30,000 & 300 & 3000 & 2500
\end{tabular}

Table 2

Size formulation for problem instances 1-5.

\begin{tabular}{llll}
\hline Instance & Continuous variables & Binary variables & Number of constraints \\
\hline 1 & 39,000 & 100 & 590 \\
2 & 44,000 & 100 & 640 \\
3 & 54,000 & 100 & 740 \\
4 & 64,000 & 100 & 840 \\
5 & 74,000 & 100 & 940 \\
\hline
\end{tabular}

Benders cycles are $0.869 \%$ and 9 respectively for both algorithms. When the algorithm hits the maximum number of Benders cycles (50), gaps provided by the Benders algorithms with cuts wcon is slightly lesser than those provided without any VI, except for problem instance 9 . This is a consequence of an upper bound slightly worse than that achieved by the Benders decomposition algorithm with VI of type wcon. To illustrate, for problem instance 4 , the upper bounds are 3,357,200 and 3,354,300 for the Benders algorithm without any VI and with VI of type wcon, respectively. For problem instance 5 , gaps provided by both algorithms are the same. Regarding lower bounds, both algorithms present the same values.

In Table 4, the lower and upper bounds, gap (\%) and number of Benders cycles are presented for Benders algorithm with VI of type wcon 1 and for the alternative Benders algorithm with Pareto optimal cuts. The Benders algorithm with VI of type wcon1 presents good results compared with the alternative Bender algorithm with Pareto optimal cuts. In some problem instances - for example, 2, 4, and 8 - gaps achieved by Benders algorithm with VI of type wcon 1 are better than those provided by the alternative Benders algorithm. However, gaps are very similar between the two algorithms. Notice that for those instances, the alternative Benders algorithm has a smaller number of Benders cycles except for problem instance 4 , where both algorithms have the same number. For problem instances $1,3,5,7$, and 9-10, gaps provided by the alternative Benders algorithm are the best. In particular for problem instance 9 , a network of $600 \times 100 \times 40$, the alternative Benders algorithm required 13 Benders cycles to achieve a gap of $0.866 \%$, while the Benders algorithm with VI of type wcon 1 required 50 Benders cycles - and hit the limit - to get a gap of $0.922 \%$. However, in Table 8 , we can observe that the smaller number of Benders cycles of the alternative Benders algorithm did not mean smaller computing times. On the contrary, the alternative Benders algorithm has the biggest computing times as a consequence of the time spending in solving the auxiliary problem to generate the Pareto optimal cuts. As observed by other authors, in our problem, the time spent to generate the optimal cuts at each iteration does not necessarily compensate with a reduction in the number of iterations and the total resolution time of the problem (Mercier \& Soumis, 2007).

Table 5 presents a summary of gaps and Benders cycles for the different Benders algorithms. To illustrate, for problem instance 9, gaps are $1.042 \%, 0.922 \%, 1.084 \%$ and $0.866 \%$ for the Benders algorithm without any VI, with VI of type wcon1, wcon and the

Table 3

Lower bound $\left(z_{l b}\right)$, upper bound $\left(z_{u b}\right)$, gap and number of Benders cycles.

\begin{tabular}{|c|c|c|c|c|c|c|c|c|}
\hline \multirow[t]{2}{*}{ Instances } & \multicolumn{4}{|c|}{ Benders algorithm without any VI } & \multicolumn{4}{|c|}{ Benders algorithm with VI wcon } \\
\hline & $z_{l b}$ & $z_{u b}$ & Gap (\%) & Benders cycles & $z_{l b}$ & $z_{u b}$ & Gap (\%) & Benders cycles \\
\hline 1 & 2457900 & 2466000 & 0.330 & 5 & 2457900 & 2466000 & 0.330 & 5 \\
\hline 2 & 2715200 & 2737200 & 0.810 & 22 & 2715200 & 2737200 & 0.810 & 22 \\
\hline 3 & 3371300 & 3400600 & 0.869 & 9 & 3371300 & 3400600 & 0.869 & 9 \\
\hline 4 & 3312700 & 3357200 & 1.343 & 50 & 3312700 & 3354300 & 1.256 & 50 \\
\hline 5 & 3314000 & 3344500 & 0.920 & 50 & 3314000 & 3344500 & 0.920 & 50 \\
\hline 6 & 4041900 & 4048550 & 0.165 & 6 & 4041900 & 4048550 & 0.165 & 4 \\
\hline 7 & 3687200 & 3720100 & 0.892 & 9 & 3687200 & 3720100 & 0.892 & 7 \\
\hline 8 & 4577300 & 4609900 & 0.712 & 7 & 4577300 & 4609900 & 0.712 & 5 \\
\hline 9 & 4077700 & 4120200 & 1.042 & 50 & 4077600 & 4121800 & 1.084 & 50 \\
\hline 10 & 4232000 & 4269000 & 0.874 & 9 & 4232000 & 4269000 & 0.874 & 8 \\
\hline
\end{tabular}

Table 4

Lower bound $\left(z_{l b}\right)$, upper bound $\left(z_{u b}\right)$, gap and number of Benders cycles.

\begin{tabular}{|c|c|c|c|c|c|c|c|c|}
\hline \multirow[t]{2}{*}{ Instances } & \multicolumn{4}{|c|}{ Benders algorithm - VI wcon1 } & \multicolumn{4}{|c|}{ Benders algorithm with Pareto optimal cuts } \\
\hline & $z_{l b}$ & $z_{u b}$ & Gap (\%) & Benders cycles & $z_{l b}$ & $z_{u b}$ & Gap (\%) & Benders cycles \\
\hline 1 & 2457900 & 2465050 & 0.291 & 2 & 2457900 & 2464300 & 0.260 & 2 \\
\hline 2 & 2715200 & 2738000 & 0.840 & 24 & 2715200 & 2738600 & 0.862 & 22 \\
\hline 3 & 3371300 & 3397800 & 0.786 & 10 & 3371300 & 3395700 & 0.724 & 12 \\
\hline 4 & 3312700 & 3355400 & 1.289 & 50 & 3312700 & 3369000 & 1.700 & 50 \\
\hline 5 & 3314000 & 3346500 & 0.981 & 21 & 3314000 & 3342000 & 0.845 & 22 \\
\hline 6 & 4041900 & 4048500 & 0.163 & 2 & 4041900 & 4048500 & 0.163 & 2 \\
\hline 7 & 3687200 & 3716600 & 0.797 & 4 & 3687200 & 3715400 & 0.765 & 4 \\
\hline 8 & 4577300 & 4598700 & 0.468 & 5 & 4577300 & 4618300 & 0.896 & 4 \\
\hline 9 & 4077700 & 4115300 & 0.922 & 50 & 4077700 & 4113000 & 0.866 & 13 \\
\hline 10 & 4232000 & 4267000 & 0.827 & 7 & 4232000 & 4258500 & 0.626 & 5 \\
\hline
\end{tabular}


Table 5

Summary gap and number of Benders cycles - different Benders decomposition algorithms.

\begin{tabular}{|c|c|c|c|c|c|c|c|c|}
\hline \multirow[t]{2}{*}{ Instances } & \multicolumn{2}{|c|}{ Without any VI } & \multicolumn{2}{|l|}{ VI wcon 1} & \multicolumn{2}{|l|}{ VI wcon } & \multicolumn{2}{|c|}{ Pareto-optimal cuts } \\
\hline & Gap (\%) & Benders cycles & Gap (\%) & Benders cycles & Gap (\%) & Benders cycles & Gap (\%) & Benders cycles \\
\hline 1 & 0.330 & 5 & 0.291 & 2 & 0.330 & 5 & 0.260 & 2 \\
\hline 2 & 0.810 & 22 & 0.840 & 24 & 0.810 & 22 & 0.862 & 22 \\
\hline 3 & 0.869 & 9 & 0.786 & 10 & 0.869 & 9 & 0.724 & 12 \\
\hline 4 & 1.343 & 50 & 1.289 & 50 & 1.256 & 50 & 1.700 & 50 \\
\hline 5 & 0.920 & 50 & 0.981 & 21 & 0.920 & 50 & 0.845 & 22 \\
\hline 6 & 0.165 & 6 & 0.163 & 2 & 0.165 & 4 & 0.163 & 2 \\
\hline 7 & 0.892 & 9 & 0.797 & 4 & 0.892 & 7 & 0.765 & 4 \\
\hline 8 & 0.712 & 7 & 0.468 & 5 & 0.712 & 5 & 0.896 & 4 \\
\hline 9 & 1.042 & 50 & 0.922 & 50 & 1.084 & 50 & 0.866 & 13 \\
\hline 10 & 0.874 & 9 & 0.827 & 7 & 0.874 & 8 & 0.626 & 5 \\
\hline Ave & 0.796 & 21.7 & 0.736 & 17.5 & 0.791 & 21 & 0.771 & 14.9 \\
\hline Max & 1.343 & 50 & 1.289 & 50 & 1.256 & 50 & 1.700 & 50 \\
\hline Min & 0.165 & 5 & 0.163 & 2 & 0.165 & 4 & 0.163 & 2 \\
\hline
\end{tabular}

Table 6

Summary of initial and last lower bound - different Benders decomposition algorithms.

\begin{tabular}{|c|c|c|c|c|c|c|c|c|}
\hline \multirow[t]{2}{*}{ \# } & \multicolumn{2}{|c|}{ Without any VI } & \multicolumn{2}{|l|}{ VI wcon 1} & \multicolumn{2}{|l|}{ VI wcon } & \multicolumn{2}{|c|}{ Pareto-optimal cuts } \\
\hline & Initial & Last & Initial & Last & Initial & Last & Initial & Last \\
\hline 1 & 2355500 & 2457900 & 2457900 & 2457900 & 2355500 & 2457900 & 2457900 & 2457900 \\
\hline 2 & 2630200 & 2715200 & 2715200 & 2715200 & 2630200 & 2715200 & 2715200 & 2715200 \\
\hline 3 & 3269800 & 3371300 & 3371300 & 3371300 & 3269800 & 3371300 & 3371300 & 3371300 \\
\hline 4 & 3227000 & 3312700 & 3312700 & 3312700 & 3230200 & 3312700 & 3312700 & 3312700 \\
\hline 5 & 3215000 & 3314000 & 3314000 & 3314000 & 3215000 & 3314000 & 3314000 & 3314000 \\
\hline 6 & 2356300 & 4041900 & 4041900 & 4041900 & 2373900 & 4041900 & 4041900 & 4041900 \\
\hline 7 & 2631200 & 3687200 & 3687200 & 3687200 & 2648200 & 3687200 & 3687200 & 3687200 \\
\hline 8 & 3271800 & 4577300 & 4577300 & 4577300 & 3288800 & 4577300 & 4577300 & 4577300 \\
\hline 9 & 3227700 & 4077700 & 4077700 & 4077700 & 3252600 & 4077600 & 4077700 & 4077700 \\
\hline 10 & 3215000 & 4232000 & 4232000 & 4232000 & 3242000 & 4232000 & 4232000 & 4232000 \\
\hline
\end{tabular}

alternative Benders algorithm with Pareto optimal cuts, respectively. For the same instance, the number of Benders cycles is 13 for the alternative Benders algorithm and 50 for all other algorithms. For problem instances 2, 4 and 8, the alternative Benders algorithm gap is worse than that obtained for the others' algorithms. In all other cases, alternative Benders algorithms perform better in term of gaps and Benders cycles, except for the problem instances mentioned above. The minimum average number of Benders cycles is obtained by the alternative Benders decomposition algorithm with Pareto optimal cuts, while the minimum average gap is obtained by the Benders algorithm with VI of type wcon 1 .

Table 6 presents the initial (iLB) and the last lower bounds $\left(z_{l b}\right)$ obtained by each Benders algorithm. Notice that the Benders algorithm with VI of type wcon1 and the alternative Benders algorithm obtain the best lower bound in the first iteration (Benders cycle) of the algorithm. Comparing the other two Benders algorithms, we observe that there is not a significant difference between the lower bounds provided by them. Table 7 shows the relative difference in percentage between the initial lower bound (iLB) and the last lower bound $\left(100 *\left[\mathrm{iLB}-z_{l b}\right] / \mathrm{iLB}\right)$ provided by the Benders algorithm (without any VI) and the Benders algorithm with VI of type wcon. This percentage represents an improvement percentage of the lower bound over the initial lower bound. Note that the maximum relative difference is $71.5 \%$ and $70.3 \%$ for the Benders algorithm and the Benders algorithm with VI of type wcon, respectively.

Table 8 reports the computational times (in seconds) required for the different Benders algorithms to obtain an integer solution within $0.9 \%$ of optimality. We observe that, in general, computational times are mostly well below $91 \mathrm{~s}$. The Benders decomposition algorithm based on Pareto-optimal cuts presents the highest computational time. This is true for problem instances $2,3,4,5$,
Table 7

Relative difference between the initial and the last lower bound provided by Benders algorithms.

\begin{tabular}{rcc}
\hline Instances & $\begin{array}{l}\text { Without any VI } \\
\text { relative difference (\%) }\end{array}$ & $\begin{array}{l}\text { VI wcon relative } \\
\text { difference (\%) }\end{array}$ \\
\hline 1 & 4.35 & 4.35 \\
2 & 3.23 & 3.23 \\
3 & 3.10 & 3.10 \\
4 & 2.63 & 2.55 \\
5 & 3.08 & 3.08 \\
6 & 71.54 & 70.26 \\
7 & 40.13 & 39.23 \\
8 & 39.90 & 39.18 \\
9 & 26.33 & 25.36 \\
10 & 31.63 & 30.54 \\
\hline
\end{tabular}

Table 8

Computational times (s) for the Benders algorithms.

\begin{tabular}{rrrrr}
\hline Instances & $\begin{array}{l}\text { Without any VI } \\
\text { time }(\mathrm{s})\end{array}$ & $\begin{array}{l}\text { VI wcon1 } \\
\text { time }(\mathrm{s})\end{array}$ & $\begin{array}{c}\text { VI wcon } \\
\text { time }(\mathrm{s})\end{array}$ & $\begin{array}{l}\text { Pareto-optimal } \\
\text { cuts time }(\mathrm{s})\end{array}$ \\
\hline 1 & 4.219 & 1.708 & 4.156 & 3.056 \\
2 & 13.853 & 14.543 & 14.096 & 30.458 \\
3 & 7.715 & 8.580 & 7.739 & 21.422 \\
4 & 35.480 & 30.628 & 31.646 & 91.731 \\
5 & 34.643 & 34.361 & 34.552 & 44.324 \\
6 & 4.797 & 1.896 & 3.244 & 3.167 \\
7 & 6.739 & 3.144 & 5.110 & 5.802 \\
8 & 6.901 & 4.390 & 4.851 & 7.462 \\
9 & 34.345 & 30.869 & 32.033 & 31.070 \\
10 & 8.354 & 5.706 & 7.276 & 10.433 \\
\hline
\end{tabular}

8 and 10 . We note that the Benders decomposition algorithm using wcon 1 cuts in general performs well in terms of computational 
times. This algorithm presents the smallest computational time for problem instances 1, 4, 5, 6, 7, 8, 9 and 10. For problem instances 2 and 3 , the Benders decomposition algorithm using wcon 1 cuts presents the worst computational time. For our problem, considering the quality of the lower and upper bounds obtained by the Benders decomposition algorithm using wcon 1 cuts and the computational time it spent in obtaining these bounds, the relative performance of this algorithm is good. Furthermore, as it was observed for other problems (Saharidis, Boile, \& Theofanis, 2011; Tang et al., 2012), a strong valid inequality, like the wcon1, can help to accelerate the convergence of the Benders algorithm by providing an improved lower bound (the best lower bound in seven out of ten, excluding Benders algorithm with Pareto-optimal cuts) and that can also eventually compensate the increase in the total resolution time produced by the poor quality of the optimality cuts generated by the Benders subproblem (without using Pareto-optimal cuts).

\section{Conclusions}

In this paper, we analyzed a reverse and sustainable supply chain network design problem. This is a NP-hard combinatorial problem and it addresses the design of a supply network that consists of three types of members: sourcing facilities (sources), reprocessing facilities, and reclamation facilities. We need to locate reprocessing facilities in order to minimize the flow cost from the origination sites to the reclamation facilities through reprocessing facilities. We proposed Benders decomposition algorithms for solving the problem. In order to accelerate the convergence of the algorithm and to improve the quality of the lower bounds, we proposed two sets of valid inequalities. One of them proved to have a significant impact on the quality of the lower bound. As a second strategy to accelerate convergence of the algorithm, we derived quasi Pareto-optimal cuts and proposed a primal based Benders decomposition algorithm for solving the problem. Both algorithms were compared and, in some problem instances, in terms of the quality of lower and upper bounds, computational results provided better performance for the primal based Benders decomposition algorithm with Pareto-optimal cuts. But in terms of total resolution time, this algorithm presents the highest values. As observed by other authors (for example, Mercier and Soumis, 2007; Sherali and Lunday, 2011), the time required to generate Pareto-optimal cuts does not necessarily offset the total resolution time. On the other hand, introducing a wcon 1 valid inequality into the Master problem from the first iteration proved to be an effective way to accelerate convergence; in addition, the first lower bound obtained by solving the Relaxed Master Problem was significantly improved. This algorithm presents the smallest computational time in eight out of ten problem instances. The final gap provided by this algorithm does not present a significance difference (less than $0.2 \%$ ) when compared with the best gap provided by the Benders algorithm with Pareto-optimal cuts. We can conclude that, for this problem, a Benders algorithm with a strong valid inequality presents a good performance in terms of gap and total resolution time.

\section{References}

Altiparmak, F., Gen, M., Lin, L., \& Paksoy, T. (2006). A genetic algorithm approach for multi-objective optimization of supply chain networks. Computers $\mathcal{E}$ Industrial Engineering, 51(1), 196-215. http://dx.doi.org/10.1016/j.cie.2006.07.011.

Alumur, S. A., Nickel, S., Saldanha-da-Gama, F., \& Verter, V. (2012). Multi-period reverse logistics network design. European Journal of Operational Research, 220(1), 67-78. http://dx.doi.org/10.1016/j.ejor.2011.12.045.

Barbosa-Povoa, A. P., Salema, M. I. G., \& Novais, A. Q. (2007). Design and planning of closed loop supply chains. In L. G. Papageorgiou \& M. C. Georgiadis (Eds.), Supply chain optimization (pp. 187-218). Wiley.
Barros, A. I., Dekker, R., \& Scholten, V. (1998). A two-level network for recycling sand: A case study. European Journal of Operational Research, 110(2), 199-214. http://dx.doi.org/10.1016/S0377-2217(98)00093-9.

Benders, J. F. (1962). Partitioning procedures for solving mixed-variables programming problems. Numerische Mathematik, 4(1), 238-252.

Bloemhof-Ruwaard, J., Salomon, M., \& Van Wassenhove, L. N. (1996). The capacitated distribution and waste disposal problem. European Journal of Operational Research, 88, 490-503. <http://www.sciencedirect.com/science/ article/pii/0377221794002118>.

Cordeau, J.-F., Pasin, F., \& Solomon, M. M. (2006). An integrated model for logistics network design. Annals of Operations Research, 144(1), 59-82. http://dx.doi.org/ 10.1007/s10479-006-0001-3.

Côté, G., \& Laughton, M. A. (1984). Large-scale mixed integer programming: Benders-type heuristics. European Journal of Operational Research, 16(3), 327-333. http://dx.doi.org/10.1016/0377-2217(84)90287-X.

Daskin, M. S., Snyder, L. V., \& Berger, R. T. (2005). Facility location in supply chain design. In A. Langevin \& D. Riopel (Eds.), Logistics systems: Design and optimization (pp. 39-65). Kluwer.

Dogan, K., \& Goetschalckx, M. (1999). A primal decomposition method for the integrated design of multi-period production-distribution systems. IIE Transactions, 31(11), 1027-1036. http://dx.doi.org/10.1080/07408179908969904.

Farahani, R. Z., \& Hekmatfar, M. (2009). Facility location: Concepts, models, algorithms and case studies. In R. Zanjirani Farahani \& M. Hekmatfar (Eds.), Media (pp. 549). Springer-Verlag. <http://www.dx.doi.org/10.1007/978-3-7908-2151-2>.

Fleischmann, Moritz, Beullens, P., Bloemhof-Ruwaard, J. M., \& Va, L. N. (2001). The impact of product recovery on logistics network design. Production and Operations Management, 10(2), 156-173.

Fleischmann, Moritz (2003). Reverse logistics network structures and design. In V. D. R. Guide \& L. N. Van Wassenhove (Eds.), Business aspects of closed-loop supply chains (pp. 117-135). New York: Carnegie Mellon University Press.

Fleischmann, Mortiz, Krikke, H. R., Dekker, R., \& Flapper, S. D. P. (2000). A characterisation of logistics networks for product recovery. Omega, 28(6), 653-666. http://dx.doi.org/10.1016/S0305-0483(00)00022-0.

Geoffrion, A., \& Graves, G. (1974). Multicommodity distribution system design by Benders decomposition. Management science, 20(5), 822-844.

Gomes, M. I., Barbosa-Povoa, A. P., \& Novais, A. Q. (2011). Modelling a recovery network for WEEE: A case study in Portugal. Waste Management (New York, NY), 31(7), 1645-1660. http://dx.doi.org/10.1016/j.wasman.2011.02.023.

Guide, V. D. R., \& Van Wassenhove, L. N. (2009). OR FORUM - The evolution of closed-loop supply chain research. Operations Research, 57(1), 10-18. http:// dx.doi.org/10.1287/opre.1080.0628.

Jayaraman, V., Guide, V. D. R., \& Srivastava, R. (1999). A closed-loop logistics model for remanufacturing. Journal of the Operational Research Society, 50(5), 497-508. http://dx.doi.org/10.1057/palgrave.jors.2600716.

Jayaraman, Vaidyanathan, Patterson, R. A., \& Rolland, E. (2003). The design of reverse distribution networks: Models and solution procedures. European Journal of Operational Research, 150(1), 128-149. http://dx.doi.org/10.1016/ S0377-2217(02)00497-6.

Krikke, H. R., Van Harten, A., \& Schuur, P. C. (1999). Business case Océ: Reverse logistic network re-design for copiers. OR Spectrum, 21(3), 381-409. http:// dx.doi.org/10.1007/s002910050095.

Le Blanc, H. M., Fleuren, H. A., \& Krikke, H. R. (2004). Redesign of a recycling system for LPG-tanks. OR Spectrum, 26(2), 283-304. http://dx.doi.org/10.1007/s00291003-0145-3.

Li, N. (2011). Research on location of remanufacturing factory based on particle swarm optimization. MSIE 2011 (pp. 1016-1019). IEEE. doi: http://dx.doi.org/ 10.1109/MSIE.2011.5707588.

Listeş, O., \& Dekker, R. (2005). A stochastic approach to a case study for product recovery network design. European Journal of Operational Research, 160(1), 268-287. http://dx.doi.org/10.1016/j.ejor.2001.12.001.

Lu, Z., \& Bostel, N. (2007). A facility location model for logistics systems including reverse flows: The case of remanufacturing activities. Computers $\&$ Operations Research, 34(2), 299-323. http://dx.doi.org/10.1016/j.cor.2005.03.002.

Magnanti, T., \& Wong, R. (1981). Accelerating Benders decomposition: Algorithmic enhancement and model selection criteria. Operations Research, 29(3), 464-484. <http://www.or.journal.informs.org/content/29/3/464.short>.

McDaniel, D., \& Devine, M. (1977). A modified Benders partitioning algorithm for mixed integer programming. Management Science, 24, 312-319.

Mercier, A., \& Soumis, F. (2007). An integrated aircraft routing, crew scheduling and flight retiming model. Computers \& Operations Research, 34(8), 2251-2265. http://dx.doi.org/10.1016/j.cor.2005.09.001.

Neto, Q. F., Walther, G., Bloemhof-Ruwaard, J. M., Van Nunen, J. A. E. E., \& Spengler, T. (2010). From closed-loop to sustainable supply chains: The WEEE case. International Journal of Production Research, 15, 4463-4481.

Papadakos, N. (2008). Practical enhancements to the Magnanti-Wong method. Operations Research Letters, 36(4), 444-449.

Rei, W., Cordeau, J.-F., Gendreau, M., \& Soriano, P. (2008). Accelerating Benders decomposition by local branching. INFORMS Journal on Computing, 21(2), 333-345. http://dx.doi.org/10.1287/ijoc.1080.0296.

Saharidis, G. K. D., Boile, M., \& Theofanis, S. (2011). Initialization of the Benders master problem using valid inequalities applied to fixed-charge network problems. Expert Systems with Applications, 38(6), 6627-6636. http:// dx.doi.org/10.1016/j.eswa.2010.11.075.

Saharidis, G. K. D., Minoux, M., \& Ierapetritou, M. G. (2010). Accelerating Benders method using covering cut bundle generation. International Transactions in Operational Research, 17(2), 221-237. 
Sahyouni, K., Savaskan, R. C., \& Daskin, M. S. (2007). A facility location model for bidirectional flows. Transportation Science, 41(4), 484-499. http://dx.doi.org/ $10.1287 /$ trsc.1070.0215.

Salema, M. I. G., Barbosa-Povoa, A. P., \& Novais, A. Q. (2010). Simultaneous design and planning of supply chains with reverse flows: A generic modelling framework. European Journal of Operational Research, 203(2), 336-349. http:// dx.doi.org/10.1016/j.ejor.2009.08.002.

Sherali, H. D., \& Lunday, B. J. (2011). On generating maximal nondominated Benders cuts. Annals of Operations Research. http://dx.doi.org/10.1007/s10479-0110883-6.

Shih, L.-H. (2001). Reverse logistics system planning for recycling electrical appliances and computers in Taiwan. Resources Conservation and Recycling, 32(1), 55-72. http://dx.doi.org/10.1016/S0921-3449(00)00098-7.

Simchi-Levi, D., Kaminsky, P., \& Simchi-Levi, E. (2007). Designing and managing the supply chain (3rd ed., Vol. 3). New York: McGraw-Hill/Irwin (pp. 354). New York: McGraw-Hill/Irwin.
Sodhi, M. S., \& Reimer, B. (2001). Models for recycling electronics end-of-life products. OR Spectrum, 23, 97-115.

Tang, L., Jiang, W., \& Saharidis, G. K. D. (2012). An improved Benders decomposition algorithm for the logistics facility location problem with capacity expansions. Annals of Operations Research. http://dx.doi.org/10.1007/ s10479-011-1050-9.

Tragantalerngsak, S., Holt, J., \& Rönnqvist, M. (2000). An exact method for the twoechelon, single-source, capacitated facility location problem. European Journal of Operational Research, 123, 473-489. <http://www.sciencedirect.com/science/ article/pii/S0377221799001058>.

Wentges, P. (1996). Accelerating Benders' decomposition for the capacitated facility location problem. Mathematical Methods of Operations Research, 44(2), 267-290.

Zhou, Y., \& Wang, S. (2008). Generic model of reverse logistics network design. Journal of Transportation Systems Engineering and Information Technology, 8(3), 71-78. http://dx.doi.org/10.1016/S1570-6672(08)60025-2. 